\title{
TURBULENT UV-LIDAR AND FIELD EXPERIMENT IN AIRPORT
}

\author{
Igor A. Razenkov", Victor A. Banakh \\ V.E. Zuev Institute of Atmospheric Optics, Tomsk, 634055, RUSSIA, *email: lidaroff@iao.ru
}

\begin{abstract}
Built the special UV (355 nm) eye safe lidar for remote detection of the atmospheric turbulence. Lidar works on the backscatter enhancement effect. Testing of lidar was carried out on the airfield at the airport Tolmachevo (Novosibirsk) in July, 2018. Sounding path was crossing the place of take-off and landing. Lidar surely fixed the position, size and intensity of turbulent zones.
\end{abstract}

\section{INTRODUCTION}

In 1972, the effect of backscatter enhancement (BSE) in the propagation of radiation in a turbulent atmosphere was discovered [1]. The effect is appeared in double propagation of radiation through the atmospheric heterogeneities of the refractive index. As a result, the average intensity of backscattered radiation on the beam's axis is increased by rays from the beam's periphery. The area in which there is an increase in intensity is small and approximately equal to the double Fresnel zone (it is equal $66 \mathrm{~mm}$ at $355 \mathrm{~nm}$ wavelength and for $3 \mathrm{~km}$ distance). Therefore, the lidar aperture should be small.

To register an enhancement in the average radiation intensity on the beam's axis, a complete coincident of the probing beam with the incoming beam is required. Herewith, the echo signal must be accumulated within a certain time interval. Practically, this is possible when probing with weak light pulses to ensure the operation of the detector and not to damage it by radiation of parasitic stray light on optics.

It is obvious that turbulent lidar is a kind of micro pulse lidar operating in the photon counting mode. Also, the turbulent lidar must have a second receiving channel (off-axis), which is not affected by turbulence. So, the normalization of the signal of the main receiving channel (on-axis) on the offaxis will eliminate the influence of a possible inhomogeneous distribution of the aerosol scattering coefficient along the sounding path [2].

For lidar to be used in special places, for example, at the airport, its radiation must be invisible and absolutely safe for the human eye. Therefore, we chose the wavelength of $355 \mathrm{~nm}$.

\section{LIDAR DESIGN}

The lidar diagram is shown in Figure 1. The receiving-transmitting telescope is located on side 2 of the optical bench (the upper part of the figure). The laser, optics and receiving box are placed on the other side 1 of the bench (the lower part of the picture). The radiation of the pulsed laser 3 by mirrors $4-9$ is directed to the collimator 10-11. Next are a half-wave plate 12 and a thinfilm polarizer 13. Mirrors 14 and 15 send the beam to the afocal telescope 17-18. In front of the telescope is a quarter wave plate 16 . Behind the telescope is a screen 19 with a pair of round holes. In Fig. 1 the on-axis channel is green and the offaxis is blue. Lidar characteristics are shown in Table. 1. Calibration procedures for turbulent lidar are detailed in [2].

\section{THEORY AND INVERSE PROBLEM}

The theory for the proposed scheme of turbulent lidar [2] was developed by Gurvich and Vorobyov [3]. Lidar equation taking into account the influence of turbulence (backscatter enhancement) can be represented as

$\frac{\left\langle P\left(x, R, \rho_{0}\right)\right\rangle}{\left\langle P_{0}\left(x, R, \rho_{0}\right)\right\rangle}=1+q\left(x, R, \rho_{0}\right)$

where $P_{0}$ is the power at the receiver in the absence of turbulence; $x$ is the distance; $\rho_{0}$ is the distance between the axes of the receivers; $R$ is the receiving aperture. The value of $q\left(x, R, \rho_{0}\right)$ is called the influence factor of turbulence on the average power of the scattered light at the receiver. 
For the case of weak and isotropic turbulence [3]

$q\left(x, R, \rho_{0}\right)=\frac{4 \pi}{R} \int_{0}^{\infty} F_{I}(\kappa, x) J_{1}(\kappa R) J_{0}\left(\kappa \rho_{0}\right) d \kappa$,

where both $J_{0}$ and $J_{l}$ are Bessel functions of zero and first order; $k_{0} \quad-$ wave number; $\Phi_{n}(\kappa, x)=0,033 C_{n}^{2}(x)|\kappa|^{-11 / 3}$ - spectrum of refractive index fluctuations; $C_{n}^{2}$ - structural characteristic of refractive index; and

$$
\begin{aligned}
F_{I}(\kappa, x) & =8 \pi k_{0}^{2} x \int_{0}^{\infty} \Phi_{n}\left[\kappa(1+\eta), \frac{x}{1+\eta}\right] \times \\
& \times\left[1-\cos \left(\frac{\kappa^{2} x}{k_{0}} \eta\right)\right] d \eta .
\end{aligned}
$$

For statistically homogeneous turbulence $\left(C_{n}^{2}=\right.$ const $)$, a good approximation of these formulas is

$$
q(x, R, 0)=\frac{0,49 C_{n}^{2} k_{0}^{7 / 6} x^{11 / 6}}{\left[1+0,4 \frac{R^{2} k_{0}}{x}\right]^{7 / 6}} .
$$
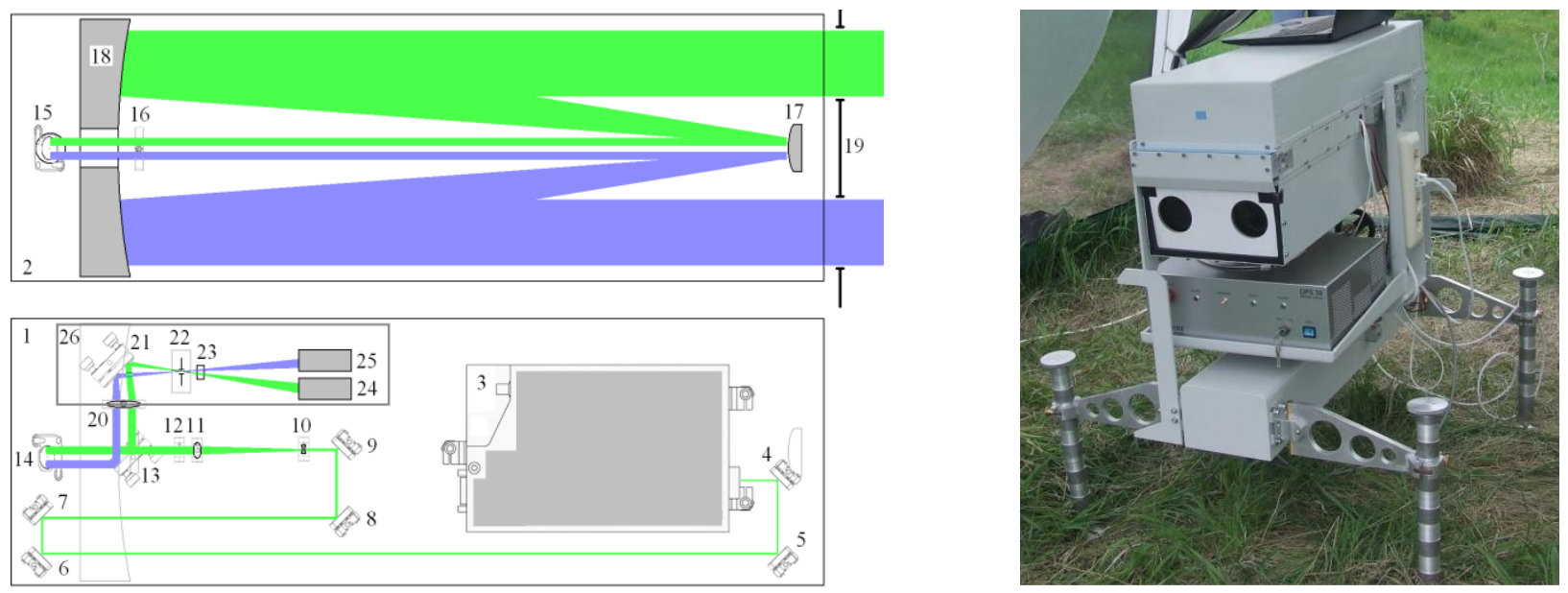

Fig. 1. Optical diagram of turbulent UV lidar (left) and real lidar on the field experiment (right).

Table 1.

\begin{tabular}{|ll|ll|}
\hline Transmitter & & Receiver & \\
\hline Laser & Solar JX 330 & Afocal telescope & Mersen \\
Wavelength & $355 \mathrm{~nm}$ & Telescope diameter & $250 \mathrm{~mm}$ \\
Pulse duration & $30 \mathrm{~ns}$ & Telescope magnification & $12,5^{\times}$ \\
Pulse repetition & $10 \mathrm{KHz}$ & Number of channels & 2 \\
Laser output & $1 \mathrm{~W}$ & Receiver aperture & $70 \mathrm{~mm}$ \\
Pulse energy & $100 \mu \mathrm{J}$ & Field of view & $300 \mu \mathrm{Rad}$ \\
Beam quality & $\mathrm{TEM}_{00}, \mathrm{M}^{2}<1,2$ & Space resolution & $15 \mathrm{~m}$ \\
Beam size $\left(\sim 1 / \mathrm{e}^{2}\right)$ & $70 \mathrm{~mm}$ & Time resolution & $>15 \mathrm{~s}$ \\
Beam divergence & $<30 \mu \mathrm{Rad}$ & PMT $(\mathrm{H} 10682-210)$ & $\mathrm{QE}=33 \%$ \\
\hline
\end{tabular}

\section{EXPERIMENT IN AIRPORT}

To test the UV turbulent lidar, the experiment was organized on the airfield of Tolmachevo airport (Novosibirsk). Lidar was located to the left of the glide path at a distance of $300 \mathrm{~m}$. The sounding path was approximately horizontal and crossed the runway at the landing point (Fig. 2).
Figure 3 schematically shows the approximate location of the (a) landing, braking and taxiing zones. And also, (b) the location of turbulent zones along the direction of sounding. The first turbulent zone appears after landing as a result of braking, the second zone occurs as a result of taxiing aircraft in the parking area. 


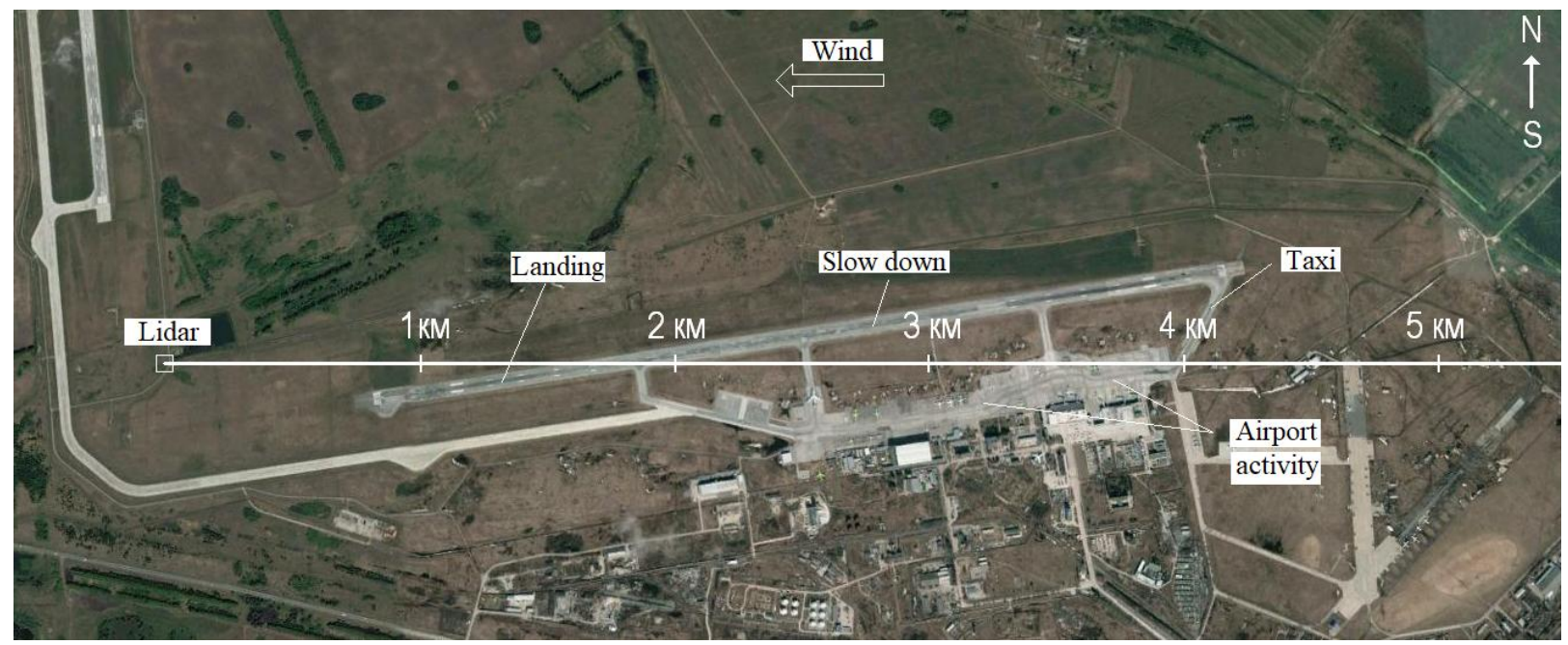

Fig. 2. Tolmachevo airport. The lidar sounding path crossing the run way at $1.5 \mathrm{~km}$. Maximum range is $5 \mathrm{~km}$.

Figure 4 shows (a) the photo of MD-11 landing and (b) touchdown of MD-11, when as a result of the wheels burning rubber an aerosol cloud appears. During the measurements the wind was from East.

Figure 5 presents examples of lidar returns. The collecting time was 1 min. In Fig. 5(a) the echo signals of the on-axis 1 and off-axis 2 receiving channels coincide, so from formula (1) it follows that there is no turbulence and $q(x)=0$. On Fig. 5(b) there is a second turbulent zone at the distance of $3.5 \mathrm{~km} . .4 \mathrm{~km}$ from the lidar. Fig. 5(c) shows the situation 2 minutes after landing MD-11 (Fig. 4). At the distance of $1300 \mathrm{~m}$ from the lidar is an aerosol cloud. The first turbulent zone is at the distance of $1500 \mathrm{~m} \ldots 1800 \mathrm{~m}$. Note, intense turbulence took place in a limited area above runway, but its influence affects the echo signal of the axial receiving channel for some time (aftereffect).

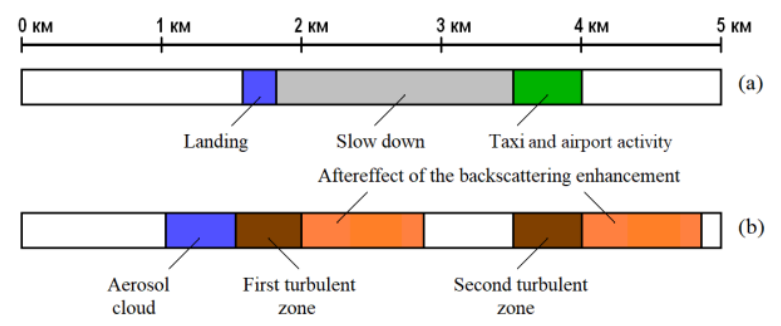

Fig. 3 Scheme of zones location: (a) landing, braking and taxiing; (b) turbulent zones and aerosol cloud.

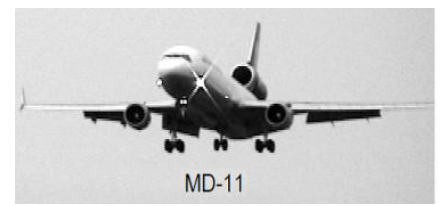

(a)

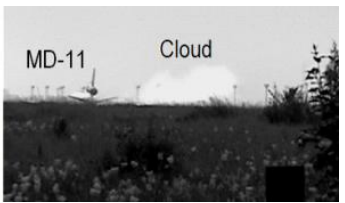

(b)

Fig. 4. Landing of the aircraft MD-11: (a) aircraft descend; (b) aircraft on the ground, aerosol cloud appearance at the moment of touching the runway.

Figure 6 presents examples of $q$-factor behavior. The first two graphs (a,b) are obtained under conditions of homogeneous turbulence $\left(C_{n}^{2}=\right.$ const $)$ at different levels of its intensity, since the values of $q$-factors differ significantly. To estimate the value of the structural characteristic of $C_{n}^{2}$, we used the formula (4), fitting the theoretical curve (dotted line) to the experimental data (circles). For the data in Fig. 6(a) and 6(b), respectively, were obtained values $C_{n}{ }^{2}=2,4 \times 10^{-15} \mathrm{~m}^{-2 / 3}$ and $C_{n}{ }^{2}=7,0 \times 10^{-14} \mathrm{~m}^{-2 / 3}$.

Figure 6(c) presents an example of an inhomogeneous distribution of the turbulence intensity along the sounding path. On Fig. 6(d) an example of superposition of the first turbulent zone on the daily turbulence intensity profile is shown. Note, the solution of the inverse problem of reconstructing the inhomogeneous profile of the structural characteristic $C_{n}^{2}(x)$ is a nontrivial problem. After landing or take-off of the aircraft, the lifetime of the turbulent zones above the runway was 2-3 minutes with size about $1 \mathrm{~km}$. 

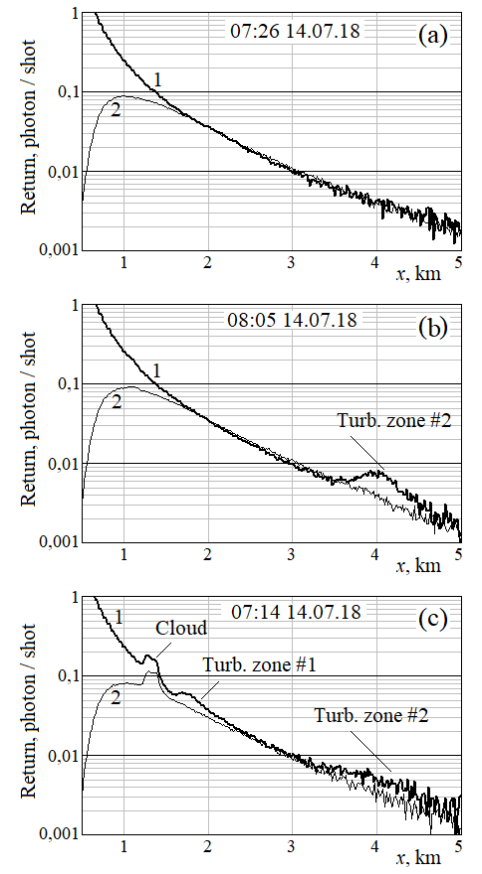

Fig. 5. Examples of returns: (a) no activity in airport; (b) the second turbulent zone is clearly recorded; (c) after landing MD-11 (see Fig. 4), there is present an aerosol cloud and both turbulent zones.

\section{CONCLUSIONS}

In Institute of Atmospheric Optics was built a turbulent UV-lidar working due to the backscatter enhancement effect. The lidar has a micro-pulse laser and an afocal telescope with a receivingtransmitting channel and a second receiving channel designed for normalization of the return of first one. Lidar implements the principle of relative measurement, which provides stability and reliability.

For the solution of the inverse problem used the Gurvich-Vorobyov theory. In conditions of homogeneous turbulence, the device allows to estimate the average value of the structural characteristic $C_{n}{ }^{2}$, proportional to the intensity of atmospheric turbulence.

Lidar was tested on the airfield at the airport Tolmachevo (Novosibirsk). Lidar allowed to register turbulent zones that occurred during landing and take-off, as well as during the taxiing, and working engines in the parking.

\section{ACKNOWLEDGMENTS}

The authors thank E.V. Gordeev, O.A. Rynkov and K.A. Rynkov for their technical support. This study was carried out according to the project of the Program for Basic Research of the Russian Academy of Sciences AAAA-A17117021310149-4.

\section{REFERENCES}

[1] A. G. Vinogradov, Yu. A. Kravtsov, and V. I. Tatarskii, "The effect of intensification of back scattering by bodies that are situated in a medium having random inhomogeneities," Radiophys. Quantum Electron. 16 (7), 818-823 (1973).

[2] I. A. Razenkov, "Turbulent lidar. I. Design," Atmos. Ocean. Opt. 31 (3), 273-280 (2018).

[3] V. V. Vorob'ev, "On the applicability of asymptotic formulas of retrieving "optical" turbulence parameters from pulse lidar sounding data: I-Equations," Atmos. Ocean. Opt. 30 (2), 156-161 (2017). 